\title{
Improving the Human Machine
}

\author{
Robotics engineers turn their sights on making humans move more \\ efficiently with the help of passive devices called exoskeletons.
}

\author{
By Michael Schirber
}

$\square$ ole Simpson calls himself a hobby runner. "I've run a couple of marathons and other long races, but I wasn't winning any medals," he says. Still, he wanted to keep up with his running buddies who are serious competitors. Being a biomechanical engineering graduate student at Stanford University, he wondered if there might be a way to "soup up" the human machine to make it run better. He and some research colleagues came up with a surprisingly simple idea: connect your ankles with a foot-long elastic strap.

Not exactly a bioengineering marvel, but the elastic worked. A controlled study showed that it reduced energy consumption during running by $6 \%[1]$. Several other research teams are working on similar devices-called exoskeletons-that can help improve walking and running efficiency. The efforts have a long history; since the late 19th century, researchers have been devising springs, hydraulics, and other mechanisms to augment human performance. "A lot of them had great ideas, but then the results didn't quite pan out the way that was expected," Simpson says.

Only within the last decade have exoskeletons demonstrated the ability to lower energy consumption. This success is partly a

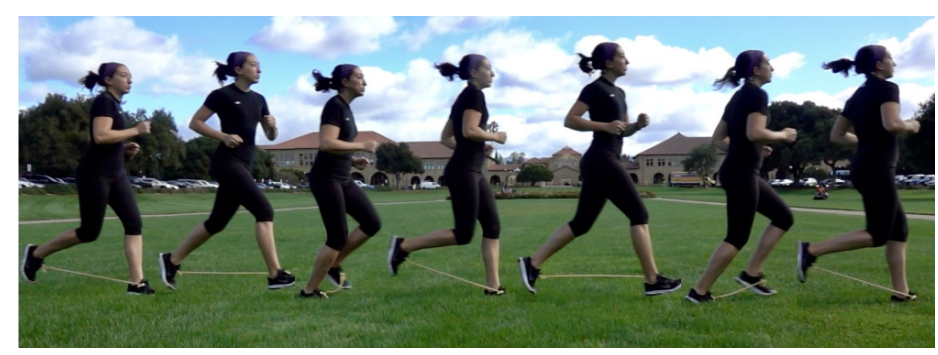

A runner demonstrates the use of an elastic strap that improves running efficiency.

Credit: C. Simpson and C. Welker/Stanford University result of advances in theoretical models of the human body in which the muscles, tendons, and joints are represented by actuators, springs, and levers. But exoskeleton developers have also realized that the human body is a unique machine that will adapt to the force inputs of a device in surprising ways. "To unlock the potential of these exoskeletons, we must understand the 'learner' connected to the device," says Jessica Selinger, a neuromechanics researcher at Queen's University in Canada who worked with Simpson on the strap.

Current research focuses on tuning the force inputs from an exoskeleton and observing the effect on a user's performance. Recent work has optimized these inputs, achieving a 15\% reduction in energy use [2]. Such devices would likely never be allowed in an official marathon race, but they might one day improve the mobility of someone who suffers from a disability or simply motivate someone to get out and run.

\section{Our Inefficiency}

As many of us can attest, jogging is a slog. The chest pounds, the gut cramps, the legs drag. It can be hard to believe that our bodies are built for this motion. And the numbers seem to back up this conclusion: only $8 \%$ of calories burned during running actually result in forward progress. "The $8 \%$ is an estimate of how much work is done against air drag at fast speeds, as this can be considered useful external work," says robotics engineer Elliot Hawkes from the University of California, Santa Barbara. A large fraction of the other $92 \%$ is wasted on braking.

To see where this braking comes in, we can think of running as a two-step process: first, the back foot pushes upward to launch the body off the ground, followed a split second later by the front foot stepping down to stop the downward fall. That stopping costs energy, as muscles in the front leg absorb the impact. This process repeats itself over and over: liftoff, 


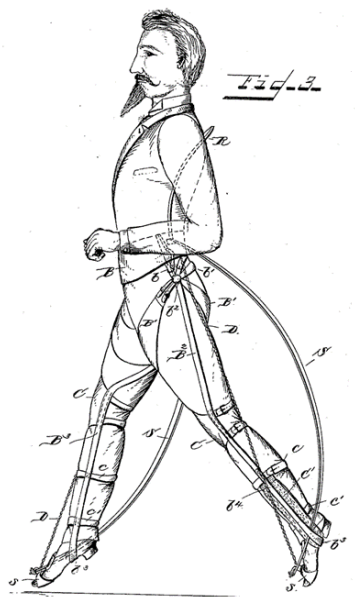

One of the earliest records of an exoskeleton is an 1890 patent for an "apparatus for facilitating walking, running, and jumping," by Nicholas Yagn.

Credit: USPTO/N. Yagn

touchdown, liftoff, touchdown, with only a small percentage of the expended energy going into forward motion.

The typical human runner expends about 3.5 joules of energy per kilogram of their body weight per meter. This "cost of transport" is comparable to other running animals in our weight class. Smaller animals typically have higher energy costs of transport than larger ones, but on a per-stride basis, it seems that all animals burn about the same amount of energy per kilogram [3]. However, humans do stand out in one regard: we excel at regulating our body temperature by sweating, which allows us to outpace horses and other running animals over distances of $20 \mathrm{~km}$ or more on hot days, Hawkes says. Some anthropologists have proposed that endurance running allowed early humans to catch and kill animals and was a key factor in our species' evolution [4].

\section{Walk Before You Run}

If we humans have been running since our earliest beginnings, is there anything that engineering can do to improve our performance? To explore this possibility, researchers have developed simplified models of human movement, such as the inverted pendulum, which describes the basics of walking.

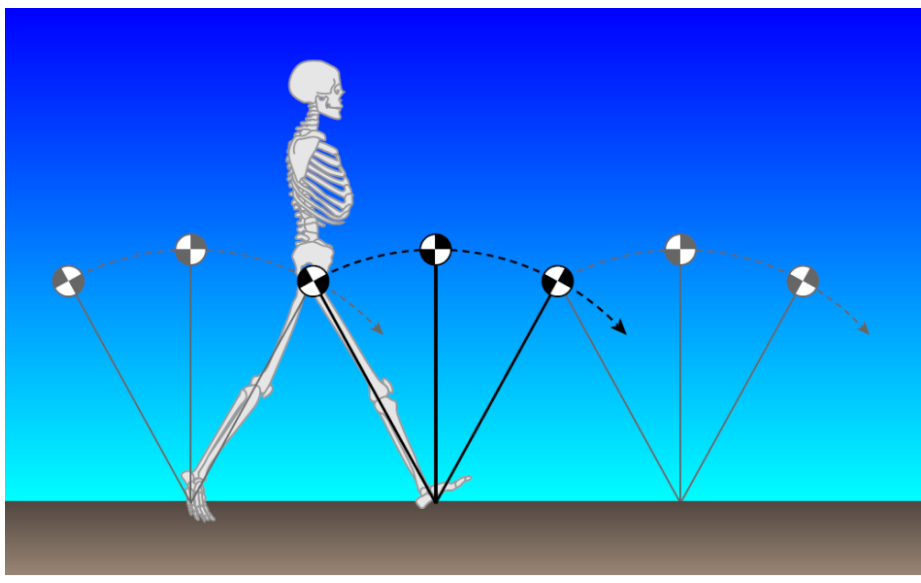

The inverted pendulum model of walking characterizes each step as the swing of a pendulum, with the front foot being the pivot point and the front leg being the beam that supports the body. With a push from the back leg, the body swings over an arc that ends when the back foot comes forward and touches down to the ground.

Credit: APS/Alan Stonebraker

To understand this model, think of how you walk: the pendulum's pivot point is your front foot on the ground, the pendulum's main mass is your body above your hip, and the beam connecting the two is your leg. Each step involves pushing off with your back leg to lift your center of mass up above your planted leg and then letting yourself fall forward until your back leg comes around in front.

"Obviously, we aren't a perfect pendulum," Selinger says. But the inverted pendulum model captures the basic features of walking, as demonstrated by the model's accurate prediction for the optimal stride frequency for humans, which is about 100 steps per minute [5]. Walking at this rate burns the fewest calories compared to faster or slower gaits, Selinger says. The typical cost of transport for walking is about 2.5 joules per kilogram per meter, which is roughly $30 \%$ less than running.

Researchers in the 1990s used the inverted pendulum model to design robots called passive walkers that could walk down an inclined plane without a power source (see video) [6]. These demonstrations showed that human walking motion is inherently stable and requires little energy input. Many robotics engineers have tried to mimic the human body in their walking 


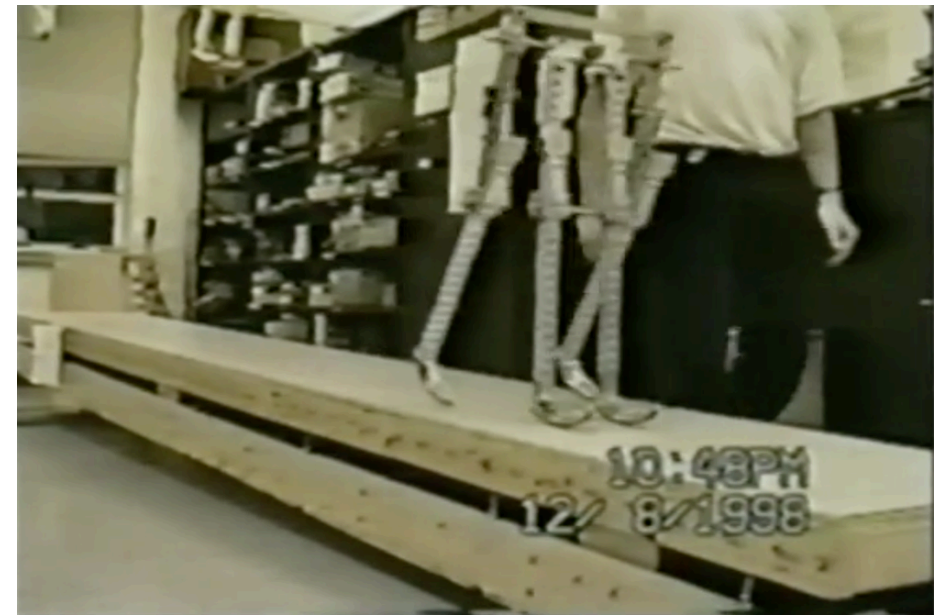

Two examples of passive dynamic walking robots developed in 1998 and 2001 in Andy Ruina's Lab at Cornell University in New York.

Credit: A. Ruina/Cornell University

machines, similar to other bio-inspired robotics development (see Flying Insects and Their Robot Imitators). But some researchers have looked in the other direction-using robotic parts to help humans move. "Robotic systems have become very efficient, so now the challenge is to improve walking and running for humans," says Rezvan Nasiri, a biomechanics expert at the University of Waterloo in Canada.

Since the early 2000s, several research groups have worked on exoskeletons that could allow a person to increase their walking endurance or help them walk while carrying a heavy weight. These devices were often metallic frames that would be attached to a user's legs and feet. Many of these exoskeletons failed, as they actually increased the energy expended by their users. "For a really long time, even though we had many, many exoskeletons that people were testing and trying, no one had actually reduced the [energy] cost below this nominal level that you would see in walking," Selinger says. Part of the problem was that some of the exoskeletons were too heavy. Another problem was uncertainty over how and when to supply extra force to the legs.

The first real success came in 2013 with an ankle exoskeleton designed by Philippe Malcolm and colleagues at Ghent

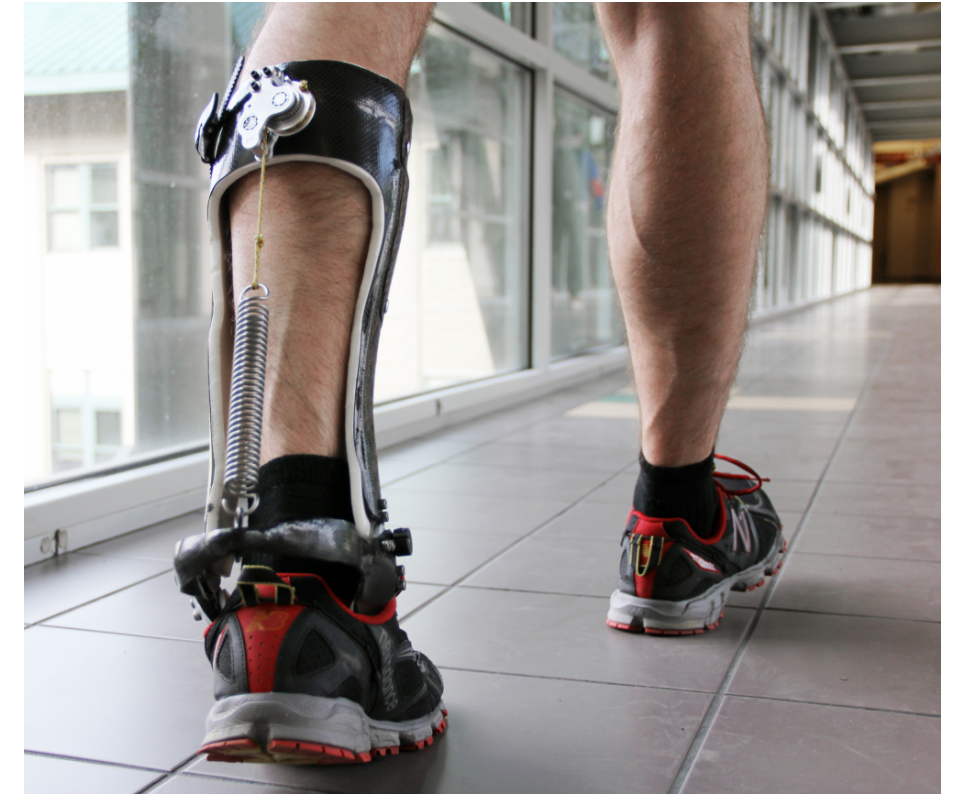

The passive ankle exoskeleton developed by Steve Collins and colleagues produced a $7 \%$ reduction in the energy cost of walking. Credit: Carnegie Mellon University

University in Belgium [7]. This device was active, which meant that a user needed to be "tethered" to a power supply. In 2015, a group led by Steve Collins, then at Carnegie Mellon University in Pennsylvania, reported on an unpowered ankle device that could be taken anywhere [8]. The exoskeleton used a ratchet and a spring to store and release energy from a person's own motion. In tests where a subject's oxygen intake was measured, the researchers determined that the metabolic cost of walking was reduced by $7 \%$.

\section{Picking up Speed}

Improving the efficiency for running has proven harder than for walking. Researchers have modeled running by modifying the inverted pendulum concept, placing a spring at the location of the foot. With this picture, one might imagine that the best way to improve running would be to give the foot more spring. And in fact, many exoskeleton designs have done just that-adding springs in parallel to the leg or attaching them to the heel of a boot.

But targeting the feet with a spring hasn't provided much benefit. "You only get out the energy that you put into the spring," says Vanderbilt University's David Braun, who studies 


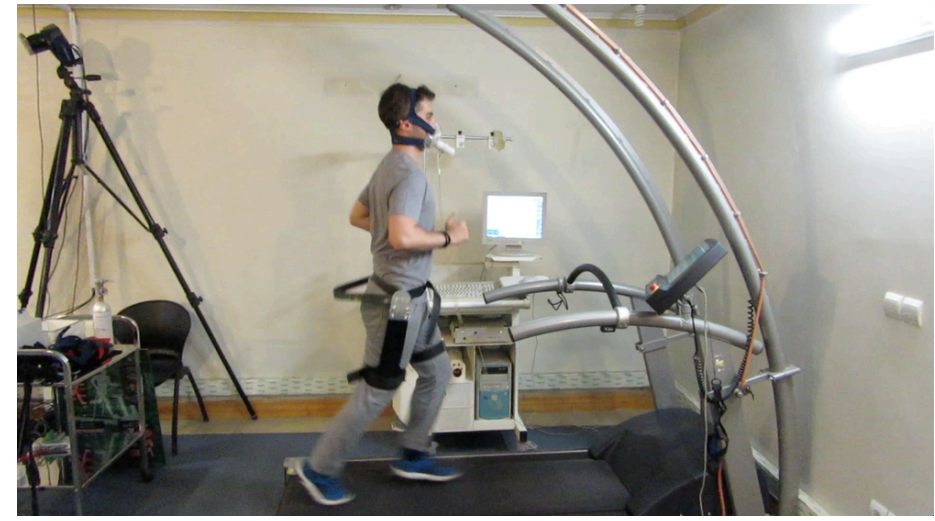

The hip-mounted exoskeleton, called I-RUN, provided energy reductions of $8 \%$ for runners on treadmills.

Credit: R. Nasiri/University of Tehran

the theoretical principles of exoskeletons. He explains that wearing a stiff spring-like that of a pogo stick-can give a boost, but you first have to compress the spring, which requires leaping high in the air for each stride. "It is unclear how this strategy would lead to a breakthrough," Braun says.

In response to the lack of success with foot-based devices, some researchers decided to target other parts of the leg. "During running, the hips are doing most of the work," Nasiri says. A large portion of that work is negative in the sense that the hip muscles must slow down each leg as it reaches the end of its swing.

To find ways to recover some of that negative work, Nasiri, working with colleagues at the University of Tehran in Iran, imagined the legs as a pair of pendulums swinging out of phase. In ordinary mechanics, one could drive this behavior with a spring connecting the pendulums. Applying this spring idea to human running, the team developed an exoskeleton, called I-RUN, that consists of a bent metal bar mounted around the back of the hips [9].

The bar acts as a spring that couples the forward swing of one leg to the backward swing of the other leg. "This spring not only reduces the muscle activity, but it can also maintain the coordination between two legs," Nasiri says. He and his colleagues tested the exoskeleton on a range of subjects-some who were athletes and others who exercised only moderately.
They computed the average metabolic rate per kilogram of body weight to be 9.2 watts without the exoskeleton and 8.5 watts with the exoskeleton, meaning the device gave an $8 \%$ reduction in the energy required to run. The team recently performed simulations to identify which leg muscles benefit-in terms of reduced fatigue-while wearing the exoskeleton [10].

\section{Accounting for Human Adaptation}

Like Nasiri's device, the strap from Simpson and his colleagues is essentially a spring connecting the legs. "It's really like tying your shoelaces together but making them elastic," Selinger says. That might sound like a recipe for disaster, but the volunteers who tried on the "exotendon," as it is called, got used to it quickly. "It makes your legs feel light and fast," says Hawkes, who worked on the project with Simpson and Selinger. "It is pretty fun."

The exotendon produced a reduction of around 6\% in energy costs during running. The efficiency improvement surprised the researchers, as leg-swinging is estimated to account for only a small fraction of energy expenditure, compared to the large energy cost of pushing off the ground and landing. However, the team showed that exotendon users were able to unlock energy savings by adapting their running motion.

The runners' main adjustment was to increase their stride frequency by about $8 \%$. Faster leg-swinging usually costs more energy, as the leg-pendulum system is driven at a frequency higher than its natural swing frequency of around 100 steps per minute. But the exotendon increases the natural frequency so that faster leg-swinging is less of an energy drain. Moreover, a faster stride is accomplished by reducing the height of each mini-jump involved in running, which cuts energy consumption.

Exotendon users were able to automatically adjust their strides based on these complex energy tradeoffs. It's as though the runner's body was saying, "okay, swinging the legs is now cheaper for me, so I'm actually going to swing my legs faster," Selinger explains.

To better understand the role of user adaptation, many biomechanics researchers are using human-in-the-loop optimization, in which human subjects wear a tunable device-one that lets researchers adjust the applied forces and torques and monitor the subject's response. "In real time you 


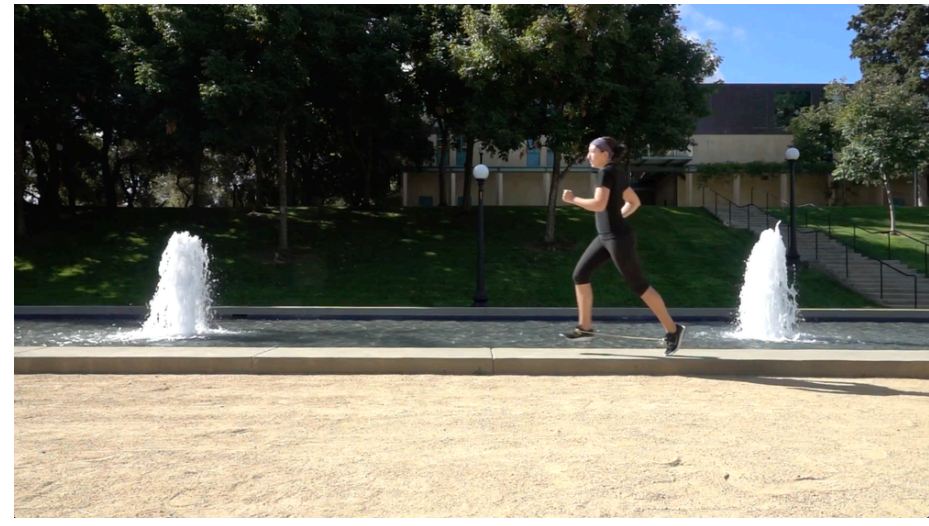

A runner demonstrating the exotendon.

Credit: C. Simpson and C. Welker/Stanford University

are tuning and adapting the parameters of the device the person is wearing to try and lower costs," Selinger says.

In a report last year, Collins' team used this approach to explore how an ankle device could improve running efficiency. Runners wore the exoskeleton on a treadmill, while computer programs varied the force inputs. They uncovered a series of force inputs that gave energy savings of $15 \%$. The trouble is that the device was not passive-it required a connection to a power supply. Several groups are developing active exoskeletons that are powered by a portable battery [11], but the extra weight lowers the energy savings, Braun says.

Collins and his colleagues did consider a spring-powered ankle device-similar to the one they used for walking-but the energy savings for runners was only $2 \%$. "We aren't really sure why the percent change was smaller than for walking," Collins says. "There are lots of reasons to expect a passive device might be more effective during running, given the spring-like behavior of the leg. But people are complicated."

\section{A Leg Up for Slow Runners}

The efficiency improvements from current exoskeletons may seem modest, but runners are often looking for any advantage they can get. A case in point is the excitement that surrounded the recent report that a new running shoe gave a $4 \%$ reduction in the energy cost of running [12].
Simpson compares the efficiency gains from an exoskeleton with those of electric bikes, which offer riders the opportunity to go further than they can with a normal bike. Exoskeletons might extend a runner's distance or help a person who has lost some mobility from age or an accident. "If you could give them any sort of benefit, maybe they could accomplish more of what they set out to do and get more out of that experience," Simpson says.

Since working on the exotendon study, Simpson has gained a new perspective on his own running. "I do like to experiment with different things, such as faster strides or lifting my feet higher, and try to feel the differences in my muscles or breathing," he says. These adjustments can help when certain muscles become sore or tired. But he admits that there's not much to do when his body has burned through all its energy. "Then I wish I was still wearing an exotendon!"

Michael Schirber is a Corresponding Editor for Physics based in Lyon, France.

\section{REFERENCES}

1. C. S. Simpson et al., "Connecting the legs with a spring improves human running economy," J. Exp. Bio. 222, jeb.202895 (2019).

2. K. A. Witte et al., "Improving the energy economy of human running with powered and unpowered ankle exoskeleton assistance," Sci. Robot. 5, eaay9108 (2020).

3. C. R. Taylor et al., "Energetics and mechanics of terrestrial locomotion. I. Metabolic energy consumption as a function of speed and body size in birds and mammals," J. Exp. Bio. 97, 1 (1982).

4. D. M. Bramble and D. E. Lieberman, "Endurance running and the evolution of Homo," Nature 432, 345 (2004).

5. K. G. Holt et al., "The force-driven harmonic oscillator as a model for human locomotion," Hum. Mov. Sci. 9,55 (1990).

6. T. McGeer, "Passive dynamic walking," Int. J. Robot. Res. 9, 62 (1990).

7. P. Malcolm et al., "A simple exoskeleton that assists plantarflexion can reduce the metabolic cost of human walking," PLoS ONE 8, e56137 (2013).

8. S. H. Collins et al., "Reducing the energy cost of human walking using an unpowered exoskeleton," Nature 522, 212 (2015).

9. R. Nasiri et al., "Reducing the energy cost of human running using an unpowered exoskeleton," IEEE Trans. Neural. Syst. 
Rehabilitation Eng. 26, 2026 (2018).

10. H. Aftabi et al., "Simulation-based biomechanical assessment of unpowered exoskeletons for running," Sci. Rep. 11, 11846 (2021).
11. G. S. Sawicki et al., J. Neuroeng. Rehabilitation 17, 25 (2020).

12. W. Hoogkamer et al., "A comparison of the energetic cost of running in marathon racing shoes," Sports Med. 48, 1009 (2017). 